\title{
Etica médica, salud y protección social*
}

\author{
Ruy Pérez-Tamayo(1)
}

\section{$\mathrm{L}$}

a invitación para preparar la Conferencia Magistral Miguel E. Bustamante, en el seno de este XI Congreso de Investigación en Salud Pública, la recibí con cierta ambivalencia, que se convirtió en franca alarma al revisar a los muy distinguidos ponentes que me han precedido en este foro y los temas de que se han ocupado, y que culminó en pánico cuando mi querido amigo, el doctor Mauricio Hernández, me envió una nota en la que decía, con su suavidad característica: "[...] nos gustaría que pudieras abordar el tema desde el punto de vista de la ética de la seguridad social [...]". Antes de recibir este mensaje yo había pensado integrar mi plática combinando dos intereses que me han ocupado en estos primeros años del siglo XXI, que son la ética médica (de la que publiqué un libro en 2002) y la historia general de la ciencia en México en el siglo $X X$ (de la que tengo un libro en prensa). Pero incluir la seguridad social como otro ingrediente más me pareció de entrada que tendría consecuencias totalmente impredecibles, por lo que decidí declinar la honrosa invitación. Pero al redactar el oficio correspondiente me llamó la atención que éste es el XI Congreso de Investigación en Salud Pública, y que su lema es: "Salud y protección social. Protección con equidad". Pensé entonces que si al tema originalmente sugerido, de "ética de la seguridad social", le agregaba el término "salud", para que se leyera como "ética, salud y seguridad social", quizá pudiera hacer algo más o menos digno de este memorable evento. Le aposté a que los organizadores que me habían hecho esta generosa invitación no se darían cuenta del sutil cambio en el título de mi plática, y gané la apuesta; de hecho, estaban tan ocupados con otros aspectos mucho más importantes del XI Congreso que (como puede verse en el programa) ni siquiera cambiaron el título de esta conferencia por el que yo había sugerido.

Como quiera que haya sido, yo voy a hablar de ética, salud y seguridad social. Mi plática se divide en las tres partes siguientes: la ética médica basada en los objetivos de la medicina; la conservación de la salud como parte de la ética médica, y las estructuras de seguridad social relacionadas con la ética médica de la salud en México. Seguramente que para muchos de ustedes estas reflexiones resultarán reiterativas y hasta aburridas, pero para mí fueron reveladoras de conexiones que hasta el momento de pensarlas y escribirlas eran insospechadas.

\section{La ética médica basada en los objetivos de la medicina}

Como estudiante de medicina en los finales de la primera mitad del siglo pasado (yo soy de la generación 43-49) nunca recibí clases de ética médica. La materia no formaba parte (y todavía no lo hace) del currículo formal de la carrera de médico cirujano y partero; a lo largo de los años las lecciones de ética médica que recibí fueron todas prácticas, no verbalizadas sino más

\footnotetext{
* Trabajo presentado en el marco de la Conferencia Magistral Miguel E. Bustamante, durante el XI Congreso de Investigación en Salud Pública, celebrado en las instalaciones del Instituto N acional de Salud Pública, en Cuernavaca, Morelos, del 2 al 4 de marzo de 2005.
}

(1) Profesor Emérito de la Universidad N acional Autónoma de México. Miembro de El Colegio N acional y de la A cademia Mexicana de la Lengua 
bien demostradas en el comportamiento cotidiano de todos aquellos de los que aprendí la profesión, o sea mis profesores, los residentes e internos con los que tuve contacto, mis compañeros estudiantes, las enfermeras y hasta las afanadoras. Nunca supe muy bien qué quería decir el término "deontología médica", y me imaginaba un código rígido y solemne, que debía seguirse sin desviación alguna, semejante a las Tablas de Moisés o a los 10 Mandamientos de la Ley de Dios. El examen profesional que hice al terminar mis estudios no incluía entonces juramento alguno, y aunque se mencionaban el Juramento Hipocrático y la entonces reciente (en 1948) Declaración de Ginebra, con su correspondiente Juramento del Médico, yo no los conocía; ni los que hablaban de ellos con gran seriedad y respeto parecían haberlos leído.

Cuando empecé a examinar en forma más sistemática los principios éticos que regulan la práctica de la medicina, los llamados "códigos de ética médica", me encontré con una lista tan larga como heterogénea. Empezando por el código del Rey Hammurabi, que data del año 1700 a.C., hasta 1803, cuando apareció el primer libro con el título de Medical Ethics, del médico inglés Thomas Percival, y a partir de entonces y hasta el día de hoy, se han promulgado docenas de documentos que pretenden enunciar los principios de ética médica, tanto a nivel regional como nacional e internacional, y tanto para una época determinada como para toda la eternidad. Hay también códigos que especifican los deberes de los médicos en general, los deberes del médico con los enfermos, los deberes de los médicos entre sí, y hasta una carta de los derechos de los pacientes. Pero que no cunda el pánico: no voy a repasar todo ese material en este momento, sino sólo a comparar dos códigos de ética médica: el más famoso de todos, el Juramento Hipocrático, que data del siglo V a.C., con uno de los más recientes y más específicamente formulados, el de la Asociación Médica Americana (AMA), en su última versión, de 2001.

Como muchos de ustedes recuerdan (lo voy a repetir para los que tengan mala memoria, porque seguramente todos ustedes lo han leído), el Juramento Hipocrático dice:

Juro por Apolo Médico, por Esculapio, por Higiene y por Panacea, y por todos los dioses y diosas, tomándolos por mis testigos, que cumpliré de acuerdo con mis capacidades y mi juicio este juramento y convenio.

Considerar al que me ha enseñado este arte igual que a mi padre y vivir mi vida en asociación con él, y si se encuentra necesitado darle parte del mío, y considerar a sus hijos como mis hermanos varones y enseñarles este arte -si desean aprenderlo- sin costo y sin com- promiso; dar una parte de mis preceptos e instrucción oral y otras formas de enseñanza a mis hijos y a los hijos del que me ha instruido y a los alumnos que han firmado el convenio y hecho el juramento de acuerdo con la ley médica, y a nadie más.

Usaré medidas dietéticas para el beneficio de los enfermos de acuerdo con mi capacidad y juicio; los protegeré del daño y de la injusticia.

No le daré una droga letal a nadie aunque la pida, ni le haré una sugestión de ese tipo. De manera semejante, no le proporcionaré un remedio abortivo a ninguna mujer. Guardaré mi arte y mi vida con pureza y santidad.

No usaré el bisturí, ni siquiera en los que sufran de la piedra, sino que me retiraré en favor de aquellos que se dedican a este trabajo.

Cualquiera que sea la casa que visite, lo haré para el beneficio del enfermo, manteniéndome alejado de toda injusticia intencional y de toda mala acción, y en especial de tener relaciones sexuales con hombres o mujeres, sean libres o esclavos.

Lo que vea o escuche en el curso del tratamiento, o aun al margen de éste, en relación con la vida de los hombres, que de ninguna manera debiera difundirse, lo mantendré en secreto y consideraré vergonzoso hablar de ello.

Si cumplo con este juramento y no lo violo, que pueda gozar de mi vida y de mi arte, honrado por la fama entre todos los hombres por todo el porvenir; pero si lo rompo y he jurado en falso, que lo opuesto sea mi suerte.

A lo largo de sus 25 siglos de vida, este Juramento se ha modificado muchas veces, para adaptarse a distintos cambios sociales, a diferentes creencias religiosas y a diversas transformaciones culturales. Dependiendo de los intereses de los distintos usuarios, a veces se han destacado las prohibiciones, otras veces la confidencialidad, y otras más el compromiso de beneficiar al enfermo; en otras ocasiones se insiste en su carácter religioso y en su compromiso con la pureza y santidad. Lo que casi nunca se subraya es el carácter excluyente de todo el que no haya tomado el juramento y firmado el convenio, el rechazo a la cirugía y la aspiración a ser "honrado por la fama entre todos los hombres por todo el porvenir", lo que es algo grandilocuente, por decirlo en forma caritativa.

El Código de Etica Médica de la AMA es representativo de muchos otros códigos contemporáneos, y dice lo siguiente:

Desde hace tiempo la profesión médica ha suscrito un cuerpo de pronunciamientos éticos desarrollados pri- 
mariamente para el beneficio del paciente. Como miembro de esta profesión, un médico debe reconocer su responsabilidad no sólo ante los pacientes sino también ante la sociedad, ante otros profesionales de la salud, y ante sí mismo. Los siguientes principios adoptados por la AMA no son leyes sino estándares de comportamiento que definen los aspectos esenciales de la conducta honorable del médico:

1. El médico estará dedicado a proporcionar servicios médicos competentes con compasión y respeto por la dignidad humana.

2. El médico debe tratar honestamente con pacientes y colegas, y exponer a aquellos médicos deficientes en carácter y competencia, o que practiquen el fraude y el engaño.

3. El médico debe respetar la ley y asumir la responsabilidad de buscar cambios en los requerimientos que sean contrarios a los mejores intereses del paciente.

4. El médico debe respetar los derechos de los pacientes, de los colegas y de otros profesionales de la salud, y también debe mantener la confidencialidad del paciente dentro de los límites de la ley.

5. El médico debe continuar estudiando, aplicando y avanzando el conocimiento científico, comunicar la información relevante al paciente, a los colegas y al público, solicitar consultas y usar el talento de otros profesionales de la salud cuando esté indicado.

6. Excepto en emergencias, el médico debe poder decidir libremente a qué pacientes atender, y el ambiente en el que desea proporcionar sus servicios.

7. El médico debe aceptar su responsabilidad de participar en actividades que contribuyan a mejorar su comunidad.

Comparando el Juramento Hipocrático con los principios de ética médica de la AMA, es obvio que no coinciden en nada: en los principios de la AMA no se convoca a ninguna deidad, no se mencionan las relaciones del médico con su profesor y su familia, no hay prohibiciones específicas relacionadas con eutanasia, aborto, relaciones sexuales o práctica de la cirugía, y el secreto profesional se condiciona a lo requerido por la ley o por el beneficio del individuo o de la comunidad. Tampoco se habla de la injusticia o de la santidad, pero en cambio se agrega que el médico está al servicio de la humanidad y no del paciente individual, que debe mejorar continuamente sus conocimientos, que su práctica médica debe ser científica, que no debe asociarse con charlatanes y que debe denunciar la conducta irregular de sus colegas.
En mi opinión, para armar un código de ética médica no conviene partir ni de una tradición antigua, ni de algún documento memorable o de un decálogo de mandamientos dogmáticos y no razonados. Como se trata de una ética profesional, lo mejor es centrarse en la profesión misma y derivar de su práctica específica los valores pertinentes y los principios de comportamiento que mejor contribuyan a alcanzar sus objetivos. Los objetivos actuales de la medicina son sólo tres: 1) preservar la salud; 2) curar, o cuando no se puede, aliviar, y siempre consolar y acompañar al enfermo, y 3) evitar las muertes prematuras e innecesarias. En el Juramento Hipocrático, los objetivos 1 y 3 no están presentes (e incluso su rechazo de la eutanasia se opone al objetivo 3), y en el código ético médico de la AMA no aparece ninguno. Pero estos tres objetivos pueden condensarse todavía más, en uno solo, que es: lograr que hombres y mujeres vivan jóvenes y sanos toda su vida y mueran lo más tarde y dignamente que sea posible.

¿Cómo puede establecerse un código de ética médica basado únicamente en los objetivos de la medicina? Mi propuesta es bien sencilla y consta de las siguientes cuatro reglas de comportamiento:

\section{Estudio continuo}

El médico tiene la obligación moral de estudiar continuamente para mantenerse al día en los avances de su profesión, con objeto de poder ofrecerle a su paciente lo mejor que existe hasta ese momento para el diagnóstico y el tratamiento de su enfermedad. Esto no es cosa fácil porque la medicina científica está avanzando y transformándose todo el tiempo; de hecho, ésta es la característica principal que la distingue de las medicinas "tradicionales", que comparadas con la primera no progresan, no aprenden de sus errores ni buscan mejorar sus resultados explorando nuevas posibilidades; sus cambios, cuando ocurren, son mucho más lentos y forman parte de las transformaciones de las culturas de las que forman parte integral. Muchos códigos de ética médica recomiendan que el médico ofrezca a sus pacientes el mejor manejo posible, mientras que otros no lo mencionan. El médico que deja de ser un estudiante de su profesión comete una grave falta de ética médica y no sólo es un mal médico y un médico malo, sino que además es un médico inmoral.

\section{Información y docencia}

El médico tiene la obligación moral de escuchar con atención, paciencia y comprensión todo lo que el paciente y sus familiares quieran decirle, y a su vez ex- 
plicarles con claridad y en lenguaje sencillo, tantas veces como sea necesario, todo lo concerniente a su enfermedad y su pronóstico, su evolución y su tratamiento. Esta es una forma importante de ser doctor, porque debemos recordar que la palabra proviene de la voz latina doscere, que significa enseñar. El médico tiene la obligación moral de enseñar su arte y su ciencia a su enfermo, a sus familiares y amigos, a sus alumnos (si los tiene) y a todos aquellos que puedan beneficiarse con su información. No hacerlo es una grave falta de ética médica, porque interfiere con el establecimiento y la conservación de una relación médico-paciente constructiva y positiva. Incidentalmente, este requerimiento de ética médica no sólo no ocurre en el Juramento Hipocrático sino todo lo contrario, pues ahí se señala que el médico sólo enseñará su arte a sus hijos, a los hijos de su maestro y a todos aquellos que hayan prestado el juramento, "y a nadie más."

\section{Investigación científica}

El médico tiene la obligación moral de contribuir a aumentar los conocimientos científicos en que se basa su profesión, con objeto de mejorar cada vez más la calidad de la atención profesional que les ofrece a sus pacientes, así como el contenido de las explicaciones que les proporciona a sus enfermos y a sus familiares, a sus estudiantes, y a todos aquellos que se beneficien de esta actividad. Por lo tanto, la investigación científica, sea básica o clínica, es también un requerimiento ético del médico, lo que significa la obligación de mantener los ojos y la mente bien abiertos a cualquier hecho novedoso o interesante, hacerse preguntas sobre lo que se observa y formular posibles respuestas para después cotejarlas con la realidad. En otras palabras, cultivar la actitud científica no sólo repitiendo lo que hacen los maestros sino intentando siempre superarlos; si este objetivo no se alcanza, si el alumno no llega a ser mejor que el maestro, la estructura educativa fracasa y el progreso es cero.

\section{Manejo integral}

El médico no debe cerrar nunca los ojos a la solicitud de ayuda del enfermo, quien acude para que lo cure o lo alivie de su padecimiento, que es algo distinto de su enfermedad, aunque este segundo término forma parte del primero. Para poner un ejemplo, el enfermo puede tener una tuberculosis pulmonar, pero lo que lo lleva a ver al médico son la astenia, la falta de apetito, la palidez, el insomnio, la febrícula, la tos, la disnea, y además el miedo a lo que pueda pasarle, a que lo tengan que operar, la angustia por su familia, por dejar de trabajar, y naturalmente el terror más o menos escondido de la muerte. Todo esto es lo que el enfermo padece, y es lo que espera que el médico le quite al curarlo. El paciente no es un caso de tuberculosis pulmonar, como seguramente sería clasificado y comentado en los corrillos médicos; el enfermo tiene un padecimiento que desde luego incluye la tuberculosis y también otras muchas cosas que forman parte de su persona, de su existencia como ser humano. Es con este carácter con el que se presenta en la consulta, y no como un par de pulmones infectados por el bacilo de Koch.

Se ha dicho mucho que la relación médico-paciente es el núcleo alrededor del que gira toda la medicina, $y$ no hay duda de que se trata de una relación crucial y de enorme importancia, pero sólo para la medicina clínica de orientación terapéutica, la que ejercían Hipócrates y los autores del Juramento Hipocrático, la que estudió y practicó uno de los más grandes médicos mexicanos del siglo XX, el doctor Ignacio Chávez, que acostumbraba referirse a la relación médico-paciente como "una confianza frente a una conciencia", y que fue la misma medicina que yo estudié en la entonces Escuela de Medicina, a partir de 1943. Pero en la segunda mitad del siglo XX, a la preocupación por el enfermo la medicina agregó su interés por conservar la salud del sujeto sano, de la comunidad sana y de la sociedad sana. A la medicina terapéutica individual se sumó la medicina profiláctica, no sólo como medidas para evitar enfermedades epidémicas (estas tienen una antigua tradición) sino como grandes proyectos multidisciplinarios médicos, económicos, políticos, culturales y sociales para preservar la salud. Como todos sabemos, ésta no es una tarea menor, algo que los médicos terapeutas puedan hacer en sus ratos libres; más bien se trata de una nueva dimensión de la medicina, de tal magnitud que requiere sus propias escuelas, $\mathrm{cu}-$ rrículos, textos, programas de posgrado, grandes proyectos de investigación, institutos magníficos como en el que estamos. Todo esto es muy reciente: recordemos que esta conferencia se da honrando la memoria de don Miguel E. Bustamante, quien se graduó de médico el mismo año en que yo nací (1924) y quien fue el primer médico mexicano doctorado en salud pública (1939).

\section{La preservación de la salud como parte de la ética médica}

Si el primer objetivo de la medicina es preservar la salud, ¿cómo se refleja en los códigos éticos de todos los tiempos? La respuesta es casi totalmente negativa: la inmensa mayoría de los códigos de ética médica no hablan de la conservación de la salud como uno de sus 
valores principales; su orientación es primariamente terapéutica. Además, a pesar de que el primer objetivo de la medicina es preservar la salud, en las cuatro reglas de la ética médica estrictamente profesional que acabo de resumir, el énfasis es la atención al enfermo. Esto puede tener su origen en dos elementos distintos: a) la incorporación formal de la salud como una de las funciones de la medicina es relativamente reciente, no tiene más de 200 años, cuando la profesión empezó a transformarse de acciones individuales basadas principalmente en la caridad, en actividades definidas como un derecho y una obligación social. Naturalmente, la higiene siempre ha formado parte del interés y la actividad de los médicos, pero para que la higiene se convirtiera en salud pública tuvieron que ocurrir cambios muy importantes en las ideas de comunidad, de sociedad, de nación y de mecanismos de cooperación internacional; b) como señala Narro:

[...] el mejoramiento de los niveles de salud de una población tiene una correlación con los resultados de las investigaciones sobre la causalidad de la enfermedad, así como en la elaboración de las medidas profilácticas $\mathrm{y}$ en menor grado de las terapéuticas.

O sea que la salud pública también se beneficia, como el resto de la medicina terapéutica, del cumplimiento de las cuatro reglas de la ética médica profesional mencionadas con anterioridad.

De todas maneras, me parece obvio que no contribuir a preservar la salud es una falta de ética médica profesional. Esto no quiere decir que todos los médicos deberían convertirse en expertos en salud pública, Dios nos libre de tal cosa. Lo que significa es que siendo la preservación de la salud uno de los objetivos de la medicina, no hacer todo lo posible para que se cumpla, en la medida de sus posibilidades y en función de sus conocimientos, es una falta de ética profesional. Un ejemplo personal me servirá para ilustrar lo que quiero decir: ¿cómo contribuye el patólogo a la preservación de la salud, tanto al nivel individual como social? Si hace bien su trabajo, al nivel individual logrará que con diagnósticos acertados los pacientes sean mejor tratados y de esa manera muchos recuperen su salud; al nivel de la población, proporcionando datos estadísticos confiables de frecuencias de distintas enfermedades en su comunidad, que en conjunto pueden servir para documentar el conocimiento epidemiológico indispensable para decidir sobre las distintas campañas de salud. El Registro Nacional de Anatomía Patológica, que ya tiene más de 20 años de estar funcionando es precisamente eso, la contribución de los médicos patólogos a la preservación de la salud, o sea al cumplimiento del primer objetivo de la medicina. Aquí deseo resaltar la figura de la doctora Patricia Alonso de Ruiz, una de las más distinguidas y justamente famosas patólogas mexicanas, quien acaba de ser reconocida con el Premio Internacional Eminent Scientist por sus excelentes contribuciones al estudio del cáncer del cuello uterino, uno de los principales problemas de salud de los países menos desarrollados, como México. Desde el punto de vista de la ética médica basada en los objetivos de la profesión, no hay duda que el excelente trabajo de la doctora Alonso de Ruiz es éticamente bueno.

\section{Las estructuras de seguridad social relacionadas con la ética médica de la salud pública en México}

En esta última parte de mi conferencia voy a referirme a un problema de gran actualidad en el mundillo médico mexicano, el de la cobertura de los servicios de salud a toda la población de nuestro país. Estoy seguro de que la mayoría de ustedes conocen este problema mucho mejor que yo, y por eso voy a evitar referirme a los distintos aspectos económicos, políticos y sociales involucrados en su planteamiento y en sus posibles soluciones (si es que las tiene). Yo voy a referirme a una sola faceta del problema, que en mis lecturas y consultas relacionadas con su planteamiento teórico y su ejecución actual surge muy pocas veces o ninguna, que es su relación con la ética médica profesional. Del compromiso del Estado por cumplir con el mandato constitucional de proporcionar servicios de salud de calidad a toda la población, de la creación del Instituto Mexicano del Seguro Social (IMSS), de la Secretaría de Salubridad y Asistencia (SSA), del Instituto de Seguridad y Servicios Sociales de los Trabajadores del Estado (ISSSTE), y ahora del Seguro Popular, sólo diré aquello que me parece relacionado con la ética médica profesional. No estoy seguro de que mi análisis les resulte interesante o hasta pertinente, dados los muchos otros aspectos del problema que no voy a mencionar. Lo que sigue tiene una sola virtud, que no es menor: la brevedad.

Empiezo con una pregunta general: ¿tiene el Estado la responsabilidad de proporcionar servicios de salud a toda la población del país? En ausencia de precisiones sobre cuál Estado, cuál nivel de responsabilidad, qué tipos de servicios de salud, y qué quiere decir "toda la población del país", una respuesta racional no es posible. Pero en la demanda de especificaciones necesarias para formularla se ha pasado por alto una relacionada con la ética médica, con los valores morales involucrados tanto en la consideración de las dis- 
tintas respuestas posibles como en la formulación final. Cuando se creó la Secretaría de Salubridad y Asistencia su mandato la responsabilizaba de los servicios de salud a todos los mexicanos, la instalación del IMSS rescató a los empleados formales y la del ISSSTE se concentró en los trabajadores del Estado, con lo que la cobertura de los servicios de salud en nuestro país quedó fijada en tres niveles: los trabajadores asalariados, al IMSS; los empleados del Estado, al ISSSTE, y el resto de la población, a la SSA.

Es obvio que desde un punto de vista ético médico, esta fragmentación de los servicios de salud entre trabajadores empleados y el resto de los mexicanos (la mayoría, campesinos no asalariados) era inaceptable. Mientras el IMSS y el ISSSTE tenían sistemas económicos que permitían una atención a la salud de un nivel de calidad razonable, la SSA se debatía entre la insuficiencia y la insolvencia. Las diferencias entre los servicios del IMSS y del ISSSTE, comparados con los de la SSA, eran reales y dolorosamente palpables. Al lado del Centro Médico del IMSS, una institución orgullo de la medicina mexicana, estaba el Hospital General de la SSA, con tantas carencias como suficiencias tenía el otro, apenas a $150 \mathrm{~m}$ de distancia. ¿Qué principio(s) ético(s) médico(s) podríamos invocar para condenarlas?

El primero en que podría pensarse es el de la equidad, que en el fondo es una expresión de la justicia social. Equidad en el acceso a servicios de salud de la más alta calidad para toda la población, al margen de edad, sexo, raza, escolaridad, ocupación, nivel económico, religión, o cualquier otra de las diferencias que se han utilizado en el pasado (y que todavía se utilizan hoy) para ejercer la discriminación entre seres humanos. La complejidad del fenómeno conocido como injusticia social o marginación es realmente enorme y puede examinarse desde muchos y muy distintos puntos de vista: histórico, político, económico, geográfico, social y otros más, sin llegar a agotarlo. En relación con la accesibilidad de distintos sectores de la población a los servicios de salud, su expresión más obvia es la gran diferencia que existe entre ellos: mientras el empleado regular tiene un seguro social (sea el Seguro Social o el ISSSTE) que incluye servicios de salud para él y su familia, el desempleado no tiene seguro alguno. Todos conocemos esta situación, y también conocemos los esfuerzos que desde 2001 se han estado haciendo a través del Seguro Popular. Pero, ¿es la falta de equidad en el acceso a los servicios de salud un problema de ética médica? ¿Somos los médicos los responsables de la estructura discriminatoria de las clases marginadas que obviamente tienen los servicios de salud del país? ¿Se nos puede acusar de falta de ética profesional? ¿O más bien el problema ético le corresponde al Estado, quien es oficialmente responsable de las políticas de salud del país?

Voy a poner un ejemplo que seguramente muchos de ustedes vivieron en otros tiempos (y que a la mejor todavía existe): me refiero al distinto tratamiento que recibían los enfermos en ciertos hospitales de la SSA, en manos de médicos que, al terminar sus ocupaciones asistenciales matutinas, se iban a sus consultorios privados a ver a sus pacientes de paga. En mis tiempos de estudiante y de interno en el Hospital General, tuve la oportunidad de comparar esos dos tipos de tratamiento: en el pabellón el enfermo no tenía ni nombre, "el cirrótico de la cama 17", nadie le explicaba qué tenía, cómo lo iban a manejar, para qué le sacaban sangre y qué medicinas le inyectaban; en cambio, en la consulta privada el mismo médico saludaba de mano a su paciente, que tenía nombre y apellido, y conversaba con él en forma por demás encantadora. Este tipo de discriminación sí me parece una falta grave de ética médica profesional, porque en el primer caso el manejo arrogante, paternalista y alejado del médico interfiere con el establecimiento de una relación médico-paciente óptima, lo que a su vez impide la realización de los objetivos de la medicina. Pero al nivel de la estructura de la sociedad, la existencia de inequidad frente a los servicios de salud de distintos sectores sociales, ¿es también responsabilidad del médico? ¿Puede calificarse como una falta de ética médica?

Confieso que no tengo una respuesta satisfactoria a ese dilema. Lunes, miércoles y viernes pienso que no, que no es una falta de ética médica, que los médicos ya tenemos bastante con la carga que nos toca dentro de nuestra profesión, para además echarnos encima la tarea de corregir los defectos de la sociedad que influyen en la salud. Pero martes, jueves y sábados pienso que sí, que la Organización Mundial de la Salud (OMS), al definir a la salud como "un estado de completo bienestar físico, mental y social, y no nada más la ausencia de enfermedad o molestias" estaba precisamente llamando la atención a los elementos no médicos (sociales) que pueden interferir con la salud, y de paso señalando que estos también deben formar parte de la preocupación de los médicos. La idea es antigua: a mediados del siglo XIX, Virchow señaló: "La medicina es una ciencia social, y la política no es otra cosa que la medicina en gran escala", y en forma menos general y más precisa, también dijo: "Los médicos son los abogados naturales de los pobres, y los problemas sociales deberían ser resueltos principalmente por ellos". Ackerknecht observa que estos dos famosos textos de Virchow revelan que su concepto era bien distinto del que tenemos hoy cuando decimos que "la 
medicina es una ciencia social". Su concepto era que "las ciencias sociales son una subdivisión de la medicina", idea muy generalizada entre los higienistas franceses de los años 1820 y que Virchow conocía bien.

Quiero terminar con una nota optimista. Para un médico con más de 50 años de ejercer y vivir la medicina en México ha sido una experiencia extraordinaria, sobre todo porque ahora que estoy empezando a ver el final del camino, puedo sentirme optimista en relación con los esfuerzos que ya se están haciendo para reducir las grandes diferencias en la asistencia a la salud en los diferentes sectores de nuestra sociedad. El problema es muy complejo y muy difícil, pero nadie dijo que fuera sencillo y fácil; lo que me satisface es que ya se ha aceptado que existe como un problema real y se está intentando resolverlo. A los críticos impacientes a los que siete años les parece un periodo demasiado largo para alcanzar la cobertura total del seguro de la salud para todos los mexicanos, les recuerdo el famoso proverbio chino que dice: "El viaje más largo siempre empieza con el primer paso".

Muchas gracias. 